\title{
Analysis of Fracture Patterns and Characteristics in Sacral Insufficiency Fracture: Do Sacral Fractures Occur in Patients Who Had Previous Lumbosacral Fusion Insufficiency Fractures or Stress Fractures?
}

\author{
Jun-Yeong Seo ${ }^{1,2}$, Kee-Yong $\mathrm{Ha}^{3}$, Young-Hoon $\mathrm{Kim}^{4}$, Hyung-Youl Park ${ }^{5}$, \\ Dong-Gune Chang ${ }^{6}$, Yong-Hwan Choi ${ }^{1}$, Young-Ho Rho ${ }^{1}$, Sang-Il Kim ${ }^{4}$ \\ ${ }^{1}$ Department of Orthopaedic Surgery, Jeju National University Hospital, Jeju National University School of Medicine, Jeju, Korea \\ ${ }^{2}$ St. Mary's Goodheal Orthopaedics, Seoul, Korea \\ ${ }^{3}$ Department of Orthopaedic Surgery, Kyung Hee University Hospital at Gangdong, Seoul, Korea \\ ${ }^{4}$ Department of Orthopedic Surgery, Seoul St. Mary's Hospital, College of Medicine, The Catholic University of Korea, Seoul, Korea \\ ${ }^{5}$ Department of Orthopaedic Surgery, Eunpyeong St. Mary's Hospital, Seoul, Korea \\ ${ }^{6}$ Department of Orthopaedic Surgery, Inje University Sanggye Paik Hospital, Inje University College of Medicine, Seoul, Korea
}

\begin{abstract}
Study Design: This study is a retrospective analysis of a case.
Purpose: The purpose of this study is to investigate the fracture patterns and risk factors of sacral insufficiency fractures (SIFs) to improve their diagnosis in clinical practice.

Overview of Literature: SIFs occur when normal stresses are applied to a bone with decreased density, most often due to osteoporosis. Individuals who receive lumbosacral fusion procedures may also suffer from increased incidents of SIFs because of decreased bone density related to aging.

Methods: In total, 55 patients with SIFs were retrospectively investigated in this study. The study population was divided into lumbosacral fusion ( $\mathrm{n}=20$ ) and non-fusion ( $\mathrm{n}=35)$ groups. Subsequently, the patients' demographic characteristics, comorbidities, medication history, results of diagnostic imaging studies, and bone mineral density were assessed. The fracture patterns were classified either according to the five typical types (H-pattern bilateral vertical plus horizontal component, unilateral vertical only, bilateral vertical only, unilateral vertical plus horizontal component, and horizontal only fracture) or atypical types.

Results: In total, 44 of 55 patients (80\%) suffered from more than one senile disease and received corresponding medications that caused secondary osteoporosis. A total of 12 patients had S1 lumbosacral fixation. Moreover, three of these 12 patients who developed a SIF immediately after a lumbosacral fracture had an unstable sacral $\mathrm{U}$ fracture. The remaining nine patients showed fracture patterns similar to the non-fusion patients. Single-photon emission computed tomography (SPECT)/computed tomography (CT) can identify fracture recurrence in previously healed fractures. In total, 24 patients (43.6\%) had fractures of the pelvis, femur, and thoracolumbar spine.

Conclusions: SIF develops in elderly patients with multiple adult diseases that can induce secondary osteoporosis. Such fractures may occur in the patients with instrumented lumbosacral fusion. Importantly, some patients showed stress fractures after multilevel instrumented lumbosacral fusion, whereas others showed insufficiency fractures. The different fracture patterns correspond to different grades of SIF, and SPECT/CT can easily identify the fracture status.
\end{abstract}

Keywords: Sacrum; Insufficiency fracture; Stress fractures; Spinal fusion; Osteoporosis

Received Sep 4, 2020; Revised Oct 19, 2020; Accepted Nov 8, 2020

Corresponding author: Sang-Il Kim

Department of Orthopedic Surgery, Seoul St. Mary's Hospital, College of Medicine, The Catholic University of Korea, 222 Banpo-daero, Seocho-gu, Seoul 06591, Korea

Tel: +82-2-2258-6775, Fax: +82-2-535-9834, E-mail: sang1kim@catholic.ac.kr 


\section{Introduction}

Sacral fractures present with a bimodal age distribution, occurring in young adults after high-energy trauma and in elderly patients with osteoporosis after low-energy trauma. The latter fractures are called sacral insufficiency fractures (SIFs) and can occur either spontaneously or after ground-level falls [1]. SIFs occur when normal stresses are applied to a bone of decreased density. Patients with SIFs often have comorbidities, such as osteoporosis, pelvic irradiation, or chronic steroid use [1-3]. The incidence of SIF is unknown because the diagnosis is frequently underrecognized $[4,5]$. However, it is apparent that SIFs are more common than their wider prevalence and have remained largely overlooked as a cause of pain and disability in the elderly and other at-risk populations [6,7]. Biomechanics also appear to play a role in the etiology of these fractures. Alterations in the lumbopelvic weight-bearing axis may also predispose individuals with spinal deformity to SIFs [8-10]. Individuals who receive lumbosacral fusion procedures may also suffer increased incidents of SIFs because of decreased bone density related to aging. The biomechanical forces applied to the sacrum in these patients differ from those in the general population [1012]. Wilde et al. [11] reported that SIFs in patients with instrumented lumbosacral fusion typically show a screwhole involvement. However, we found that not all SIFs in the lumbosacral fusion involved screw holes. Therefore, we have investigated the fracture patterns in the patients with instrumented lumbosacral fusion and in those with no history of lumbosacral fusion. Additionally, we surveyed the risk factors associated with the development of SIFs to improve the diagnosis of SIFs in clinical practice.

\section{Materials and Methods}

This retrospective case series was approved by the institutional review boards of the two participating institutions (Seoul St. Mary's Hospital IRB approval no., KC17RESI0730; Jeju University Hospital IRB approval no., 201603-004-002). The requirement for informed consent from patients was omitted because of the retrospective design of this study. The study population comprised patients with SIFs between June 2010 and December 2016. A SIF was diagnosed based on physical and imaging assessments. The inclusion criteria were: sacral fracture caused by minor trauma, such as ground-level fall or weight- lifting, or even in the absence of trauma. The exclusion criteria were: pathological fractures caused by metastasis or infection and high-energy trauma, such as fall from a height or a traffic accident. Finally, 55 patients treated for SIFs (two men and 53 women) were enrolled in this study. In total, 31 patients were treated at institution 1, and 24 patients were treated at institution 2. A total of 20 patients had previous instrumented lumbosacral fusion (fusion group) and 35 patients had no previous fusion (non-fusion group). Patients underwent plain radiography and at least one of the following examinations: magnetic resonance imaging, computed tomography (CT), bone scintigraphy, and/or single-photon emission computed tomography (SPECT/CT). The SPECT/CT data were acquired between 2 and 4 hours after the intravenous injection of approximately $9 \mathrm{MBq} / \mathrm{kg}$ of 99mTc-MDP (Fujifilm RI Pharma Co. Ltd., Tokyo, Japan) at institution 1, and 15-20 mCi (555-740 MBq) of 99mTc-HDP (Mallinckrodt Medical Inc., St. Louis, MO, USA) at institution 2 on a Symbia T6 dual-headed gamma camera (Siemens SAS Medical Solutions, Munich, Germany). Additionally, accompanying fractures of the pelvis, femur, and/or vertebrae were also surveyed in this study.

\section{Risk factors of sacral insufficiency fracture}

Age, sex, body mass index (BMI), mechanism of trauma, and pathological conditions of the lower extremities were investigated in this study. Comorbid diseases and medications were determined based on the medical records to identify the risk factors for SIF. Comorbidities were assessed by Charlson comorbidity index (CCI) [13]. Cancer history and radiation therapy history were also examined. Bone mineral density (BMD) of the femoral neck $\left(\mathrm{g} / \mathrm{cm}^{2}\right)$ and T-scores were measured by dual-energy $\mathrm{X}$-ray absorptiometry. Medication history for the treatment of osteoporosis was also investigated in this study. In the lumbosacral fusion group, we investigated the fusion level, the lowest instrumented vertebra (LIV), whether the fracture line extended to the screw hole, presence or absence of iliac screw fixation, and the interval from fusion to fracture.

\section{Fracture pattern of sacral insufficiency fracture}

SIFs were classified as either typical type (H-pattern of bilateral vertical plus horizontal component, unilateral 

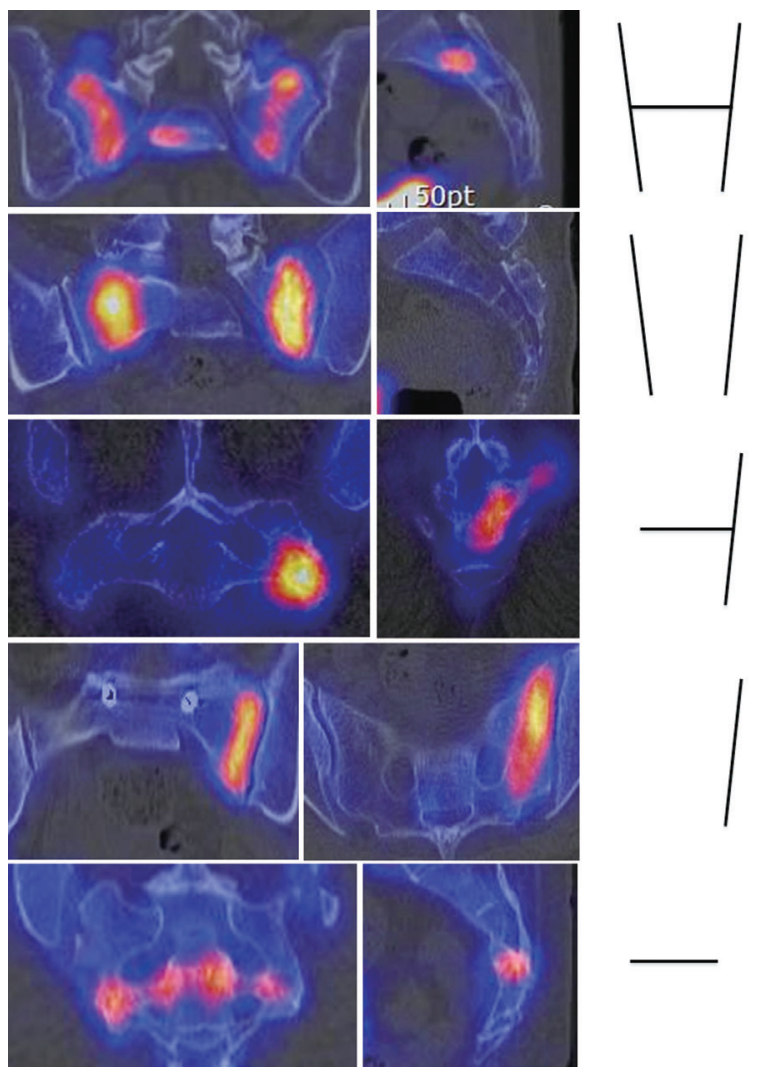

Fig. 1. Single photon emission computed tomography showing the typical types of sacral insufficiency fracture: $\mathrm{H}$-pattern bilateral vertical plus horizontal component, unilateral vertical only, bilateral vertical only, unilateral vertical plus horizontal component, and horizontal only fracture (superior to inferior). vertical only, bilateral vertical only, unilateral vertical plus horizontal component, and horizontal only fracture) (Fig. 1) or atypical type (sacral $U$ fracture with spinopelvic instability, promontory compression fracture) (Fig. 2). Importantly, the correlation between unilateral fracture and lower extremity pathology was also assessed in the groups.

\section{Statistical analysis}

The independent samples $t$-test and chi-square test were performed to compare the lumbosacral fusion and nonfusion groups. Analyses were performed using SPSS software ver. 14.0 (SPSS Inc., Chicago, IL, USA). The results are expressed as means \pm standard deviation. In all the analyses, $p<0.05$ was used to indicate statistical significance.

\section{Results}

\section{Patients and their comorbidities}

The mean age at diagnosis of sacral fracture was $73.5 \pm 9.6$ years (range, $52-90$ years). The mean BMI $(\mathrm{n}=47)$ was $23.0 \pm 3.9 \mathrm{~kg} / \mathrm{m}^{2}$. The mean BMD $(\mathrm{n}=47)$ of the femoral

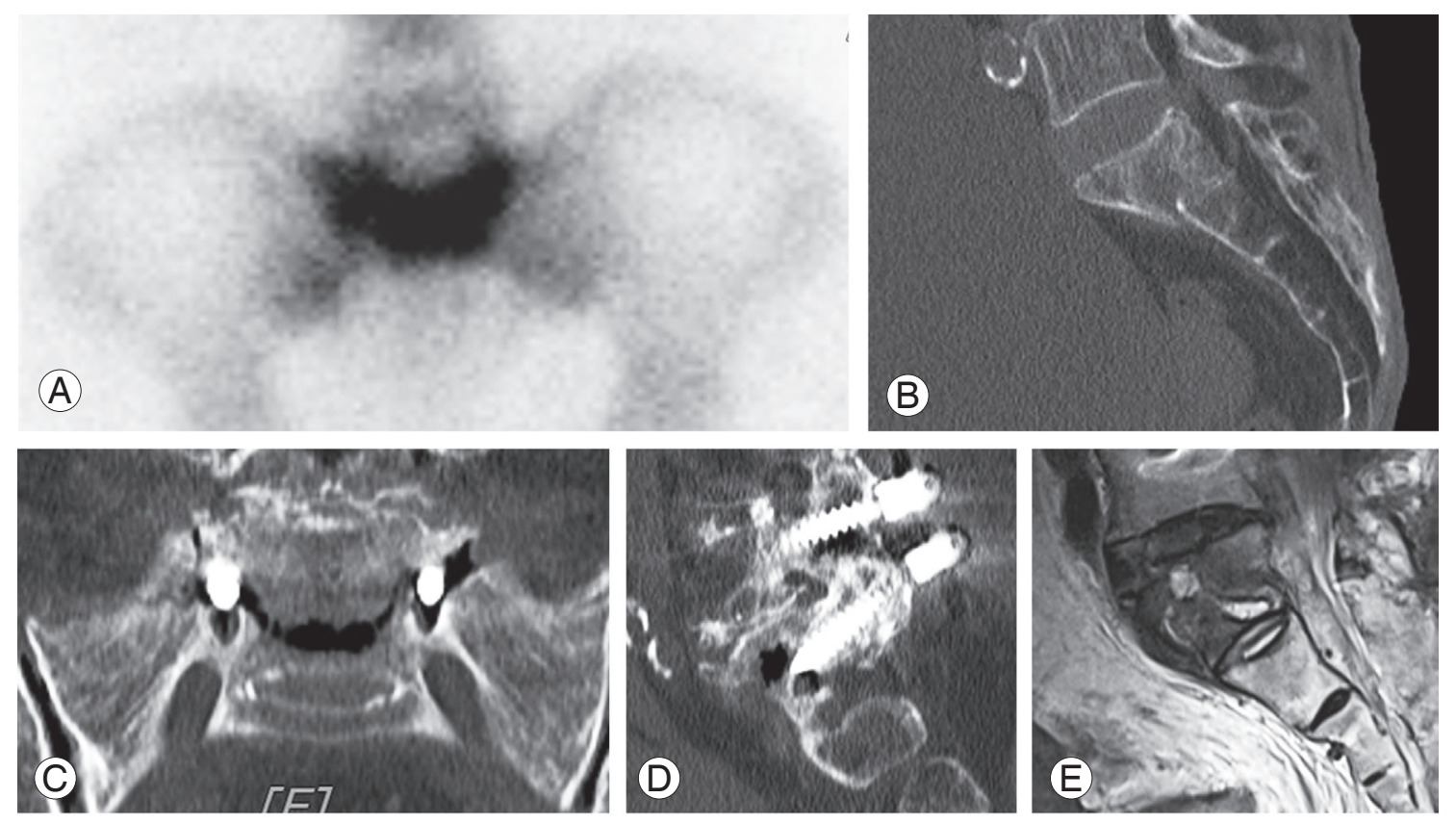

Fig. 2. Atypical types of sacral insufficiency fracture. (A, B) The top two images show promontory fracture of the sacrum. (C-E) The bottom three images show sacral U fracture with spinopelvic instability. 
Table 1. Comparison of demographic data between the lumbosacral fusion and non-fusion groups

\begin{tabular}{|c|c|c|c|}
\hline Characteristic & Fusion group ( $\mathrm{n}=20$ ) & Non-fusion group ( $\mathrm{n}=35)$ & $p$-value \\
\hline Age (yr) & $68.8 \pm 9.3$ & $76.2 \pm 8.8$ & 0.005 \\
\hline Sex (female, \%) & 100.0 & 94.3 & 0.53 \\
\hline Body mass index $\left(\mathrm{kg} / \mathrm{m}^{2}\right)$ & $24.8 \pm 3.3$ & $21.9 \pm 3.9$ & 0.013 \\
\hline \multicolumn{4}{|c|}{ Dual-energy X-ray absorptiometry (hip) } \\
\hline Bone mineral density $\left(\mathrm{g} / \mathrm{cm}^{2}\right)$ & $0.69 \pm 0.11$ & $0.55 \pm 0.10$ & $<0.001$ \\
\hline T-score & $-2.7 \pm 0.6$ & $-3.1 \pm 0.9$ & 0.048 \\
\hline Osteoporosis medication & & & 0.58 \\
\hline Previous medication & 12 & 17 & \\
\hline No history of medication & 8 & 18 & \\
\hline
\end{tabular}

Values are presented as mean \pm standard deviation or number, unless otherwise stated.

neck was $0.6 \pm 0.1 \mathrm{~g} / \mathrm{cm}^{2}$, and the mean T-score of the femoral neck was $-3.0 \pm 0.8$. All the patients included in this study could walk independently before the episode of SIFs. Patients in the fusion group (68.8 \pm 9.3 years) were younger than those in the non-fusion group $(76.2 \pm 8.8$ years) $(p=0.005)$. Additionally, BMI was higher in the fusion group $\left(24.8 \pm 3.3 \mathrm{~kg} / \mathrm{m}^{2}\right)$ than in the non-fusion group $\left(21.9 \pm 3.9 \mathrm{~kg} / \mathrm{m}^{2}\right)(p=0.013)$. The mean BMD of the femur neck was significantly higher in the fusion group than in the non-fusion group. However, there were no differences in sex distribution and the history of osteoporosis medication (Table 1).

Among the total study population of 55 patients, 11 had no disease history. Table 2 summarizes the prevalence of comorbid conditions. The mean CCI were $1.2 \pm 1.4$ and $1.1 \pm 1.3$ in the fusion and non-fusion groups $(p=0.81$ ), respectively. In total, 44 patients (80\%) suffered from more than one disease, and 28 of these patients had more than two diseases. In total, 32 patients had hypertension and were receiving antihypertensive drugs for treatment. A total of eight patients had cardiovascular diseases other than hypertension, and 10 patients suffered from diabetes mellitus. In all, nine patients had problems related to the central nervous system, and eight patients had a history of cancer, but none had a history of radiation therapy of the pelvic area. In total, seven patients had problems in the liver and gastrointestinal tract, five patients suffered from rheumatologic disease, five others had a history of long-term steroid treatment, and four patients had kidney problems.
Table 2. Prevalence of comorbidities in each category of the $\mathrm{CCl}$

\begin{tabular}{lcc} 
Comorbid conditions & $\begin{array}{c}\text { Fusion } \\
\text { group }(\mathrm{n}=20)\end{array}$ & $\begin{array}{c}\text { Non-fusion } \\
\text { group }(\mathrm{n}=35)\end{array}$ \\
\hline Myocardial infarction & $3(15)$ & $1(2.9)$ \\
\hline Congestive heart failure & $1(5)$ & $3(8.6)$ \\
\hline Peripheral vascular disease & 0 & $1(2.9)$ \\
\hline Cerebrovascular disease & $3(15)$ & $2(5.7)$ \\
\hline Chronic pulmonary disease & $1(5)$ & $3(8.6)$ \\
\hline Rheumatic disease & $2(10)$ & $3(8.6)$ \\
\hline Peptic ulcer disease & 0 & $1(2.9)$ \\
\hline Mild liver disease & 0 & $2(5.7)$ \\
\hline Diabetes without chronic complications & $6(30)$ & $3(8.6)$ \\
\hline Diabetes with chronic complications & 0 & $1(2.9)$ \\
\hline Renal disease & $1(5)$ & $1(2.9)$ \\
\hline Any malignancy without metastasis & $3(15)$ & $4(11.4)$ \\
\hline Moderate or severe liver disease & 0 & $2(5.7)$ \\
\hline Mean CCl) & $1.2 \pm 1.4(0-4)$ & $1.1 \pm 1.2(0-4)$ \\
\hline
\end{tabular}

Values are presented as number (\%) or mean \pm standard deviation (range). $\mathrm{CCl}$, Charlson comorbidity index.

${ }^{\text {al }} p$-value was 0.81 on the Student $t$-test.

\section{Can osteoporosis treatment affect sacral insufficiency fracture?}

In total, 29 patients had taken medication for osteoporosis; 17 of these patients were in the non-fusion group (16 patients had been treated with bisphosphonate and one had received raloxifene) and 12 were in the fusion group (11 had been treated with bisphosphonate and one had received raloxifene) ( $p=0.58$ ). The mean periods of osteoporosis medication were $3.5 \pm 3.1$ years and $8.1 \pm 5.0$ years in 
Table 3. Comparison of sacral insufficiency fractures between the lumbosacral fusion and non-fusion groups

\begin{tabular}{lccc} 
Fracture pattern & $\begin{array}{c}\text { Fusion } \\
\text { group }(\mathrm{n}=20)\end{array}$ & $\begin{array}{c}\text { Non-fusion } \\
\text { group }(\mathrm{n}=35)\end{array}$ & $p$-value \\
\hline H-sign & 8 & 23 & 0.02 \\
\hline Bilateral vertical only & 0 & 2 & \\
\hline Unilateral vertical only & 4 & 1 & \\
Unilateral vertical and horizontal & 2 & 4 & \\
\hline Horizontal only & 3 & 2 & \\
\hline Promontory & 0 & 3 & \\
\hline Sacral U fracture & 3 & 0 \\
\hline
\end{tabular}

Table 4. Numbers of patients according to fusion level by the LIV

\begin{tabular}{lcccc} 
Fusion level & LIV $=S 1$ & LIV=L5 & LIV $=\mathbf{L} 4$ & Total \\
\hline 1 & 1 & 4 & & 5 \\
2 & $1(1)$ & 2 & & 3 \\
3 & 3 & 1 & & 4 \\
4 & $2\left(1^{a}\right)$ & & & 2 \\
5 & 2 & & 1 & 2 \\
7 & $2\left(2^{\left.a^{a}\right)}\right.$ & & & 3 \\
9 & 1 & & 1 & 20 \\
\hline Total & 12 & 7 & & 1 \\
\hline
\end{tabular}

The numbers in the blank denote cases with screw-hole involvement. LIV, lowest instrumented vertebra.

alIndicate patients with sacral U fracture, corresponding to spinopelvic dissociation.

Table 5. Comparison between sacral insufficiency fracture patients whose LIV was S1 (LIV=S1) and those whose LIV was L5 and/or upper (LIV $\geq L 5)$

\begin{tabular}{|c|c|c|c|}
\hline Variable & LIV=S1 (n=12) & $\operatorname{LIV} \geq \operatorname{L5}(\mathrm{n}=8)$ & $p$-value \\
\hline \multicolumn{4}{|l|}{ Age (yr) } \\
\hline $50-59$ & 2 & 3 & 0.54 \\
\hline $60-69$ & 3 & 1 & \\
\hline $70-79$ & 6 & 4 & \\
\hline$\geq 80$ & 1 & 0 & \\
\hline \multicolumn{4}{|l|}{ Body mass index $\left(\mathrm{kg} / \mathrm{m}^{2}\right)$} \\
\hline Under-weight (<18.5) & 0 & 1 & 0.47 \\
\hline Normal (18.5-24.9) & 5 & 4 & \\
\hline Over-weight (25.0-29.9) & 5 & 2 & \\
\hline \multicolumn{4}{|l|}{ Dual-energy $X$-ray absorptiometry } \\
\hline Bone mineral density $\left(\mathrm{g} / \mathrm{cm}^{2}\right)$ & $0.71 \pm 0.11$ & $0.67 \pm 0.12$ & 0.49 \\
\hline T-score & $-2.78 \pm 0.66$ & $-2.59 \pm 0.33$ & 0.42 \\
\hline Screw hole involvement & 4 & 0 & NA \\
\hline
\end{tabular}

Values are presented as number or mean \pm standard deviation.

LIV, lowest instrumented vertebra; NA, not applicable. the non-fusion and fusion groups ( $p=0.024)$, respectively.

\section{Can lumbosacral fixation increase stress on the sacrum?}

Table 3 shows a comparison between patients with and without a history of lumbosacral fusion. Fracture patterns of SIF were significantly different between the fusion and non-fusion groups $(p=0.02)$. In the lumbosacral fusion group ( $\mathrm{n}=20)$, one-level fusion was performed in five patients, two-level fusion in three patients, three-level fusion in four patients, four-level fusion in two patients, five-level fusion in two patients, seven-level fusion in three patients, and nine-level fusion in one patient (Table 4). The mean interval from fusion to a SIF was $98.0 \pm 74.7$ months. Sacral fixation was performed in 12 patients (LIV=S1), whereas seven patients had lumbar fusion to L5 caudally (LIV=L5). Moreover, one patient had fusion to L4 (LIV=L4) (Table 4). There were no differences with respect to age, BMI, or BMD between the patients with LIV=S1 and patients with LIV $\geq$ L5 (Table 5).

Three patients developed sacral fracture immediately

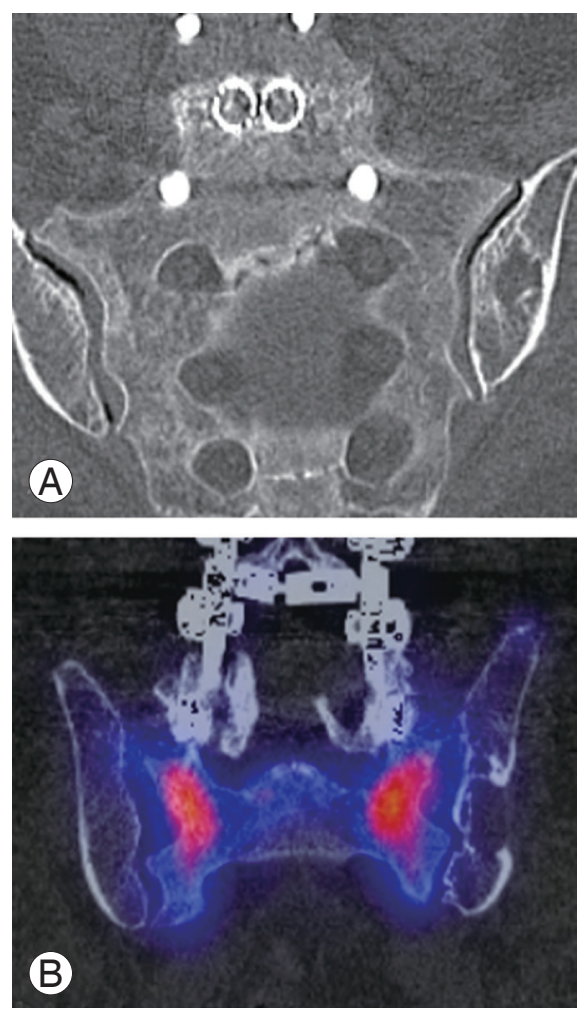

Fig. 3. A 73-year-old female patient who received L4-S1 instrumented fusion 10 years ago. (A) Computed tomography (CT) showed an old horizontal fracture with calluses and sclerotic changes in the sacrum. (B) Single photon emission CT showed an acute bilateral vertical only fracture. 

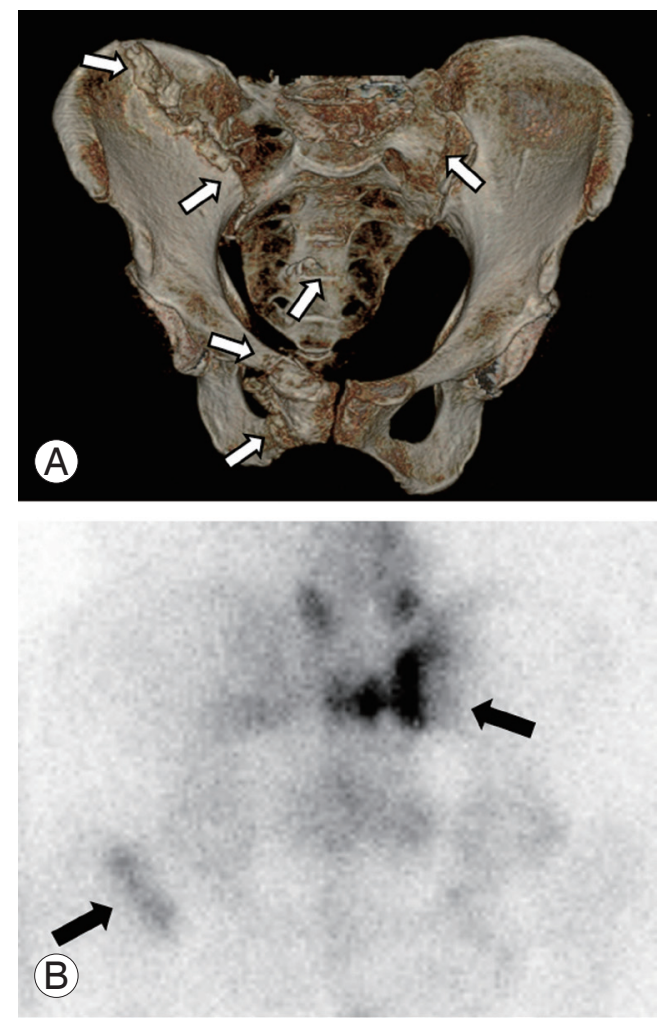

Fig. 4. (A) Pelvic computed tomography shows right ilium, pubis, and ischium fractures with a left-sided unilateral sacral alar and horizontal fracture (arrows). (B) Bone scan shows a unilateral sacral alar and horizontal fracture accompanied by a contralateral femoral neck fracture (arrows).

after lumbosacral fixation. In these cases, the fracture line extended to the S1 screw and the fracture pattern was of a sacral $\mathrm{U}$ fracture, which presented as spinopelvic dissociation (Fig. 2). They had no visible fracture line during the surgery. All these patients were allowed to ambulate with orthosis on the second day after surgery. The first patient presented back pain on postoperative 14 days, the second patient presented pain on postoperative 17 days, and the third patient presented back pain at postoperative 4 weeks. These three cases of SIF seemed to suffer stress fractures at the caudal end of multilevel instrumented lumbosacral fusion.

\section{Sacral insufficiency fractures can progress to weak bone on weight bearing}

Three patients showed different stages of SIF in the pelvis and/or hip. One patient with right-sided unilateral alar fracture progressed to a classic $\mathrm{H}$ fracture 2 weeks later. Another patient showed an old fracture line horizontally on CT but acute bilateral vertical-only fracture was identified on SPECT/CT (Fig. 3). The third patient who had a classic H-type SIF with the fracture of both superior rami showed bilateral hip fracture 3 months later.

\section{Fracture pattern identified by nuclear imaging}

The most common fracture pattern was bilateral alar fracture with a horizontal component, which is known as the classic $\mathrm{H}$-sign (58\%). Other patterns of fracture were distributed evenly in the lumbosacral fusion and non-fusion groups. SPECT/CT could discriminate new fractures during the healing process (Fig. 3). The classic $\mathrm{H}$-sign fracture occurred more frequently in the non-fusion group than in the fusion group ( $p=0.02$ ) (Table 3 ).

\section{Characteristics of unilateral sacral alar fractures}

Unilateral sacral alar fractures were found in 11 patients (right, $n=3$; left, $n=8$ ). Three of these patients had accompanying injury of the pelvis and femur (two patients had accompanying contralateral pelvic bone fracture, whereas one patient had contralateral femur neck fracture) (Fig. 4). With regard to the lower extremity pathology (total of 15 patients), five of 11 patients with unilateral SIF had lower extremity issues: two patients had bilateral total knee arthroplasty (TKA), two others had unilateral TKA (one on the contralateral side, the other on the same side), and one patient had previous bipolar hip hemiarthroplasty on the contralateral side.

\section{Accompanying axial bone fractures}

Accompanying fractures developed in 24 patients (43.6\%) in the pelvis, femur, and thoracolumbar spine. Among them, 16 patients (29.1\%) had pelvic fracture, usually of the superior and inferior rami, acetabulum, or ilium. In total, 13 patients had vertebral fractures, two patients had femur fractures (one of the femur neck and one intertrochanteric fracture), two patients had rib fractures, and seven patients had fractures in more than two areas.

\section{Discussion}

In this study, patients who developed a SIF had several chronic diseases that caused frailty, and both the disease itself and its medications had negative effects on bone 
tabolism. Hypertension was the most common comorbid disease. It is difficult to conclude definitively that hypertension is a risk factor for SIFs. However, osteoporosis and hypertension share similarities in etiopathology, genetic basis, and the effect of various nongenetic factors $[14,15]$. The most relevant etiological factors for both diseases are low calcium intake and level, vitamin D and vitamin $\mathrm{K}$ deficiency, high sodium salt consumption, and low or very high nitric oxide levels [16]. The patients in this study had been taking many kinds of antihypertensive drugs that can affect bone metabolism. Further investigations are required to determine the positive and negative effects of antihypertensive drugs on bone [16]. In addition to hypertension, most of our patients suffered from agingrelated diseases, such as diabetes mellitus, liver disease, chronic kidney disease, etc. Not only the diseases themselves, but also the medications used for their treatment can result in the development of secondary osteoporosis. Most of the patients were postmenopausal women who had taken osteoporosis medication for short periods, which can lead to a vicious cycle of severe osteoporosis.

Sacrum function is important in the pelvic outlet, in which the orientation of the sacrum relative to the ilium is present such that the axial forces lock the sacrum into the pelvic ring and further stabilize the sacroiliac articulation $[17,18]$. A few studies have reported the morphological classification of SIFs $[19,20]$. The mechanism of development of characteristic H-type pattern of SIF has been explained as a vertical fracture originating from the lateral position on the sagittal plane, followed by the loss of support for the central sacrum resulting in a horizontal fracture. The upper body weight longitudinally transfers downward to the central portion of the sacral body, resulting in a lateral sacral alar fracture [19]. Lumbosacral lordosis produces an anteroinferior vector of force, which causes the cortical breakage of the anterior sacral body $[19,21]$. This concept was first proposed by Cooper et al. [22]. The different scintigraphic patterns correspond to different grades of severity of sacral trauma. Bone scans may show progression from the initial uptake in a single sacral alar to the characteristic $\mathrm{H}$-shaped pattern of increased uptake [23]. The three subtypes of typical fractures, namely unilateral vertical only, bilateral vertical only, and unilateral vertical plus horizontal component, are considered while developing classic $\mathrm{H}$-type SIFs, and patients had a better prognosis than classic H-type SIF. The horizontal only fracture had better prognostic out- comes than the other types because both the vertical supports of sacral alar are spared. In case with long lumbosacral fusion, the spinopelvic dissociation fracture had the worst prognosis, thus requiring revisional surgery. When lumbopelvic fixation was performed, the upper body weight was transferred through the instrument. Then, the sacrum was preserved from the stress concentration.

Unilateral sacral fractures have been associated with the abnormalities of the lower extremities, such as hip or knee pathology, which would alter gait and place stress on the sacrum $[19,24]$. In our study, unilateral sacral alar fractures were identified in 11 patients, among whom three had accompanying contralateral pelvis or femur neck fractures that were not regarded as true unilateral SIFs. The enrolled patients were elderly and therefore had osteoarthritic changes in the lower extremities and some biomechanical problems that were associated with the lower extremities. Asymmetrical forces on standing or walking may give rise to fractures in the osteoporotic sacrum.

Wilde et al. [11] reported that SIF in patients with lumbosacral fusion tends to show screw-hole involvement. In their study, all of 23 sacral fractures occurred horizontally through the sacral body and involved the screw holes. In our study, eight of 12 patients with LIV=S1 status had sacral fractures distant from the sacral screw holes. Only four patients developed SIF through the screw holes, three of whom developed sacral $U$ fractures that subsequently required spinopelvic stabilization. These three cases had the involvement of multiple levels (>four levels) and fractures developed within 6 weeks, similar to the results reported by Wilde et al. [11]. In their report, all the 23 patients underwent interbody fusion at the L5-S1. However, only eight of the 12 patients in our study underwent L5-S1 interbody fusion. The surgical strategies were also different from ours; they performed L5-S1 interbody fusion anteriorly or posteriorly, and all but one patient with anterior interbody fusion underwent posterior lumbar interbody fusion at the L5-S1. The authors believe that these different surgical strategies would result in the different fracture patterns of SIFs.

Patients in the lumbosacral fusion group readily developed SIF at a younger age than those in the non-fusion group. BMI was not correlated with lumbosacral fusion. The mean BMD of the femoral neck was significantly greater in the fusion group as compared to the non-fusion group. The bone quality or quantity of the sacrum may be 
affected by lumbosacral fusion, even in the cases where LIV is L5 or S1. Stress shielding and osteopenia around the sacrum can occur adjacent to the instrumented vertebra [25]. In a successfully instrumented spine fusion, the resorption of osteophytes and sclerosis have been identified and significant bone loss or resorption can occur with the removal of the external stress caused by fusion [26]. The bone quality of the sacrum may be altered by lumbosacral fusion. Further biomechanical studies are needed to investigate osteoporosis in the sacrum around the sites of lumbosacral fixation.

We identified $44 \%$ of accompanying fractures on the axial skeleton, among which $29 \%$ developed accompanying pelvic fracture, especially on the pubis or ischium. We adopted the term "pelvic insufficiency fracture" in this report, as used previously $[27,28]$. The incidence of pelvic fracture accompanying SIF is high due to the use of the pelvis instead of the sacrum for body weight bearing [28]. In our study, three patients with a unilateral sacral alar fracture showed bilateral pelvic insufficiency fractures, with the fracture line transmitting bilaterally. Linstrom et al. [19], in a finite element analysis of walking, suggested several locations, including the acetabular region, pubic rami, ischial rami, and inner curve of the iliac wing, could be responsible for pelvic insufficiency fracture because of high load bearing $[28,29]$. We encountered a typical case confirming their hypothesis, thereby showing pelvic insufficiency fracture and bilateral intertrochanteric fractures in all these locations.

Bone scintigraphy is one of the most sensitive techniques for the detection of SIFs. Moreover, this technique can help in identifying the accompanying fractures. SPECT/CT directly correlates bone scintigraphy findings with anatomical structures via three-dimensional reconstructions [30]. Additionally, SIFs are not a single-episode phenomenon, instead it manifests as an ongoing process of collapse. SPECT/CT can easily identify newly developing fractures during the healing process.

This study had some limitations. First, it used a retrospective design and the diagnostic modality differed among individuals. However, SIFs are commonly misdiagnosed or missed entirely, because their presentation is similar to other spinal pathologies. Additionally, SIFs do not occur in isolation; additional fractures may develop in the pelvis, hip, or spine, as described previously. Therefore, in this study, different diagnostic tools were used to find other hidden pathologies in the lumbar spine, pel- vis, and hip joint. Second, the BMD of the lumbar spine was not analyzed in this study because it could not be checked in the patients with lumbosacral fusion. Third, the number of the subjects was small and all the populations were not investigated regardless of the development of SIFs. Therefore, we could not analyze the incidence of SIFs. Fourth, the patients with previous fusion surgery underwent different surgeries in terms of the fusion levels, surgical methods, and surgeons. These factors would have had a different biomechanical effect on the occurrence of SIFs.

\section{Conclusions}

SIFs readily develop in the elderly patients with accompanying multiple adult diseases that induce secondary osteoporosis. Patients with lumbosacral fusion may show either stress fractures because of multilevel instrumentation or insufficiency fractures. SIFs should be suspected in the elderly patients with acute back or buttock pain, because they are not uncommon and thus can be included in the differential diagnosis. Importantly, SPECT/CT is a useful tool to identify the precise location of a SIF.

\section{Conflict of Interest}

No potential conflict of interest relevant to this article was reported.

\section{Acknowledgments}

This work was supported by a research grant from Jeju National University Hospital in 2017.

\section{Author Contributions}

JYS and KYH designed the study and wrote the manuscript. YHK and HYP analyzed the data. DGC, YHC, and YHR collected the data. SIK revised the manuscript.

\section{References}

1. Saraux A, Valls I, Guedes C, Baron D, Le Goff P. Insufficiency fractures of the sacrum in elderly subjects. Rev Rhum Engl Ed 1995;62:582-6.

2. Moreno A, Clemente J, Crespo C, et al. Pelvic insufficiency fractures in patients with pelvic irradiation. 
Int J Radiat Oncol Biol Phys 1999;44:61-6.

3. Cho M, Moon SH, Lee JH, Lee JH. Investigation of comorbidity, trauma history, and osteoporotic fractures in the postmenopausal population: a nationwide, observational, and cross-sectional study of Korean orthopedic outpatient clinics. Asian Spine J 2019;13:779-85.

4. Jacquot JM, Finiels H, Fardjad S, Belhassen S, Leroux JL, Pelissier J. Neurological complications in insufficiency fractures of the sacrum. Three case-reports. Rev Rhum Engl Ed 1999;66:109-14.

5. Sudhir G, K L K, Acharya S, Chahal R. Sacral insufficiency fractures mimicking lumbar spine pathology. Asian Spine J 2016;10:558-64.

6. Tsiridis E, Upadhyay N, Giannoudis PV. Sacral insufficiency fractures: current concepts of management. Osteoporos Int 2006;17:1716-25.

7. Weber M, Hasler P, Gerber H. Insufficiency fractures of the sacrum: twenty cases and review of the literature. Spine (Phila Pa 1976) 1993;18:2507-12.

8. Gotis-Graham I, McGuigan L, Diamond T, et al. Sacral insufficiency fractures in the elderly. J Bone Joint Surg Br 1994;76:882-6.

9. Lourie H. Spontaneous osteoporotic fracture of the sacrum: an unrecognized syndrome of the elderly. JAMA 1982;248:715-7.

10. Decker S, Lafage R, Krettek C, et al. Is sacral extension a risk factor for early proximal junctional kyphosis in adult spinal deformity surgery? Asian Spine J 2020;14:212-9.

11. Wilde GE, Miller TT, Schneider R, Girardi FP. Sacral fractures after lumbosacral fusion: a characteristic fracture pattern. AJR Am J Roentgenol 2011;197:184-8.

12. Nakashima H, Kanemura T, Satake K, et al. The prevalence and risk factors for S2 alar-iliac screw loosening with a minimum 2-year follow-up. Asian Spine J 2020;14:177-84.

13. Charlson ME, Pompei P, Ales KL, MacKenzie CR. A new method of classifying prognostic comorbidity in longitudinal studies: development and validation. J Chronic Dis 1987;40:373-83.

14. Yang S, Nguyen ND, Center JR, Eisman JA, Nguyen TV. Association between hypertension and fragility fracture: a longitudinal study. Osteoporos Int 2014;25:97-103.

15. Varenna M, Manara M, Galli L, Binelli L, Zucchi F, Sinigaglia L. The association between osteoporosis and hypertension: the role of a low dairy intake. Calcif Tissue Int 2013;93:86-92.

16. Ilic K, Obradovic N, Vujasinovic-Stupar N. The relationship among hypertension, antihypertensive medications, and osteoporosis: a narrative review. Calcif Tissue Int 2013;92:217-27.

17. Garfin SR, Eismont FJ, Bell GR, Fischgrund JS, Bono CM. Rothman-Simeone and Herkowitz's the spine. 7th ed. Philadelphia (PA): Elsevier Inc.; 2018.

18. Dunsker SB. The unstable spine: thoracic, lumbar, and sacral regions. Orlando (FL): Grune \& Stratton; 1986.

19. Linstrom NJ, Heiserman JE, Kortman KE, et al. Anatomical and biomechanical analyses of the unique and consistent locations of sacral insufficiency fractures. Spine (Phila Pa 1976) 2009;34:309-15.

20. Bakker G, Hattingen J, Stuetzer H, Isenberg J. Sacral insufficiency fractures: how to classify? J Korean Neurosurg Soc 2018;61:258-66.

21. McFarland EG, Giangarra C. Sacral stress fractures in athletes. Clin Orthop Relat Res 1996;(329):240-3.

22. Cooper KL, Beabout JW, Swee RG. Insufficiency fractures of the sacrum. Radiology 1985;156:15-20.

23. Jones DN, Wycherley AG. Bone scan demonstration of progression of sacral insufficiency stress fracture. Australas Radiol 1994;38:148-50.

24. Schneider R, Yacovone J, Ghelman B. Unsuspected sacral fractures: detection by radionuclide bone scanning. AJR Am J Roentgenol 1985;144:337-41.

25. Wood KB, Schendel MJ, Ogilvie JW, Braun J, Major MC, Malcom JR. Effect of sacral and iliac instrumentation on strains in the pelvis: a biomechanical study. Spine (Phila Pa 1976) 1996;21:1185-91.

26. Ha KY, Molon JN, Ahn JH, Kim YH. Fate of osteophytes and sclerosis in fused segments after lumbar fusion. Spine (Phila Pa 1976) 2014;39:E1110-5.

27. Clamp JA, King RJ, O'Hara JT, Hahn DM. Osteoporotic pelvic insufficiency fracture with gross instability. J Trauma 2008;64:1380-2.

28. Peh WC, Khong PL, Yin Y, et al. Imaging of pelvic insufficiency fractures. Radiographics 1996;16:335-48.

29. Chary-Valckenaere I, Blum A, Pere P, Grigon B, Pourel J, Gaucher A. Insufficiency fractures of the ilium. Rev Rhum Engl Ed 1997;64:542-8.

30. Al-faham Z, Rydberg JN, Oliver Wong CY. Use of SPECT/CT with 99mTc-MDP bone scintigraphy to diagnose sacral insufficiency fracture. J Nucl Med Technol 2014;42:240-1. 